\title{
INVARIANT VECTOR FIELDS AND GROUPOIDS
}

\author{
EUGENE LERMAN
}

\begin{abstract}
We use the notion of isomorphism between two invariant vector fields to shed new light on the issue of linearization of an invariant vector field near a relative equilibrium. We argue that the notion is useful in understanding the passage from the space of invariant vector fields in a tube around a group orbit to the space invariant vector fields on a slice to the orbit. The notion comes from Hepworth's study of vector fields on stacks.
\end{abstract}

\section{CONTENTS}

1. Introduction

2. Isomorphic vector fields and their flows 4

3. Local normal form for an invariant vector field 6

4. Genericity in the space of invariant vector fields 11

5. Relation with the work of Hepworth and Baez-Crans 13

References

\section{INTRODUCTION}

Dynamics and bifurcation theory of group-invariant vector fields is an old and well-established area of mathematics. The literature on the subject is vast, and we will not attempt to review it. The area draws on a number of fields that include representation theory, invariant theory, transformation groups, singularity theory, equivariant transversality and geometric theory of dynamical systems to name a few.

The goal of this paper is to add category theory to the arsenal of tools. More specifically we'd like to bring to the attention of the dynamics community the notion of isomorphism of invariant vector fields and to show that it is useful and natural. The source of the idea lies in Hepworth's study of vector fields on stacks [4].

Traditionally one considers the collection $\Gamma(T M)^{G}$ of vector fields on a manifold $M$ invariant under an action of a Lie group $G$ as a vector space with a topology, which is often a Whitney $C^{\infty}$ topology. Note that multiplication by scalars in not continuous [7, pp. 46-47] in these topologies, so $\Gamma(T M)^{G}$ is a semi-topological vector space. ${ }^{1}$ On the other hand Hepworth (op. cit.) tells us that the space $\Gamma(T M)^{G}$ of invariant vector fields is naturally the set of objects of a groupoid. In this paper we combine the two approaches by considering the space of invariant vector fields as the space of objects in a linear semi-topological groupoid which we denote by $\mathbb{X}(G \times M \rightrightarrows M)$ (see Definition 5.1). This said, we strive to keep the paper accessible to readers unfamiliar with groupoids and formulate most of our results without mentioning groupoids explicitly. We hope that a reader can ignore all the references to groupoids and still profit from reading the paper.

We give two applications of viewing invariant vector fields as objects of a groupoid to equivariant dynamics. The first one quantifies non-uniqueness of linearization of invariant vector fields near relative equilibria; we thus revisit the work of Krupa [8]. The second deals with the notions of genericity for invariant vector fields.

\footnotetext{
${ }^{1}$ The term "semi-topological vector space" is rarely used but it is convenient for our purposes. It is a also a somewhat ambiguous - it is also used to describe vector spaces with a topology for which multiplication by scalars is continuous but addition is not. We trust that our use of the term will not cause any undue confusion.
} 
Given a manifold $M$ with a proper action of a Lie group $G$, consider a $G$-invariant vector field $X \in \Gamma(T M)^{G}$. Recall that a point $x \in M$ is a relative equilibrium of $X$ if the vector $X(x)$ is tangent to the orbit $G \cdot x$. Let $H$ denote the stabilizer of $x$. Following Krupa choose a slice $S$ through $x$ to the action of $G$. Then the restriction of $X$ to the slice $S$ can be decomposed as

$$
\left.X\right|_{S}=X^{\mathrm{h}}+X^{S}
$$

where $X^{S}$ is an $H$-invariant vector field on the slice and $X^{\mathrm{h}}$ is tangent to the $G$-orbits (see also Lemma 8.5.3 in [5]). Note that $X^{S}$ vanishes at $x$. One can then deduce a number of useful results about the dynamics of $X$ by studying the dynamics of $X^{S}$ in a neighborhood of its equilibrium $x$. Similarly, given a family of vector fields $X_{\lambda}$ one can analyze the bifurcations of a relative equilibrium of $X_{\lambda}$ in terms of the bifurcation of its projection $X_{\lambda}^{S}$ onto the slice. For all practical purposes one may think of the slice $S$ as a vector space with a linear action of the compact group $H$. We note that dynamics and bifurcation theory of invariant vector fields on representations have been studied intensely and extensively, and is well understood. So reducing the study of invariant vector fields near relative equilibria to the study of zeros of invariant vector fields in representations is a natural thing to do.

However, neither the slice $S$ nor the decomposition (1.1) for a given choice of a slice are unique. Nor is it clear that if a vector field $X$ is generic then its projection $X^{S}$ is generic and conversely. Thus given two different choices of slices $S, S^{\prime}$ through $x$ and $x^{\prime} \in G \cdot x$ respectively and two choices of splittings (1.1), it is far from clear how exactly the vector fields $X^{S}$ and $X^{S^{\prime}}$ are related (if they are related at all). In particular there is no apparent relation between the spectra of the linearizations $D X^{S}(x)$ and $D X^{S^{\prime}}\left(x^{\prime}\right)$. Consequently the notion of the spectrum of a vector field at a relative equilibrium does not seem to make sense (it is known the real part of the spectrum is well-defined; see [5, Lemma 8.5.2]). This is one instance where the notion of isomorphism of vector fields turns out to be useful.

Given an action of a Lie group $G$ on a manifold $M$, as before, consider the vector space

$$
C^{\infty}(M, \mathfrak{g})^{G}=\{\psi: M \rightarrow \mathfrak{g} \mid \psi(g \cdot m)=A d(g) \psi(m) \quad \text { for all } m \in M, g \in G\}
$$

of Lie algebra valued equivariant maps. This vector space maps into the space of invariant vector fields: we have a linear map

$$
\partial: C^{\infty}(M, \mathfrak{g})^{G} \rightarrow \Gamma(T M)^{G}, \quad \partial(\psi):=\psi_{M}
$$

where the vector field $\psi_{M}$ is defined by

$$
\psi_{M}(m):=\left.\frac{d}{d t}\right|_{t=0} \exp (t \psi(m)) \cdot m \quad \text { for all } m \in M .
$$

Following Hepworth (cf. [4, Proposition 6.1]) we have:

Definition 1.5 (Isomorphic vector fields). Two invariant vector fields $X, Y \in \Gamma(T M)^{G}$ are isomorphic if there is an equivariant map $\psi \in C^{\infty}(M, \mathfrak{g})^{G}$ with

$$
X=Y+\psi_{M},
$$

where $\psi_{M}$ is defined by (1.4) above.

The flows of two isomorphic vector fields are related by time-dependent "gauge transformation." More precisely in Section 2 below we prove:

Theorem 1.6. Suppose two $G$-invariant vector fields $X$ and $Y$ on a manifold $M$ are isomorphic in the sense of Definition 1.5. Then there exists a family of maps $\left\{F_{t}: M \rightarrow G\right\}$ depending smoothly on $t$ so that flows $\Phi_{t}^{X}, \Phi_{t}^{Y}$ of $X$ and $Y$ respectively satisfy

$$
\Phi_{t}^{X}(m)=F_{t}(m) \cdot \Phi_{t}^{Y}(m)
$$

for all $(t, m) \in \mathbb{R} \times M$ for which $\Phi_{t}^{X}(m)$ is defined. 
The purpose of Theorem 1.6 is (1) to give readers a feel for what it means dynamically for two vector fields to be isomorphic and (2) to show that the notion of equivalence of vector fields has been around implicitly in equivariant dynamics literature for quite some time. We then show that isomorphic vector fields occur naturally and that the notion of isomorphism of vector fields is useful.

Theorem 1.7. Let $X \in \Gamma(T M)^{G}$ be an invariant vector field, $x_{1}, x_{2}$ two points in $M$ on the same orbit, $S_{1}, S_{2}$ slices through the points $x_{1}, x_{2}$ respectively and $X^{S_{i}} \in \Gamma\left(T S_{i}\right)^{H_{i}}$ the components of $X$ tangent to the corresponding slices $\left(H_{i}\right.$ is the stabilizer of $\left.x_{i}\right)$. Then, shrinking the slices if necessary, there exists an equivariant diffeomorphism $\varphi: S_{1} \rightarrow S_{2}$ so that the vector fields $\varphi_{*}\left(X^{S_{1}}\right)$ and $X^{S_{2}}$ are isomorphic.

Remark 1.8. The decomposition (1.1) implicitly used in the statement of Theorem 1.7 is different from the one defined by Krupa: instead of using an invariant Riemannian metric on $M$ we use a left invariant connections on the principal bundles $G \rightarrow G \cdot x_{i}, i=1,2$.

Theorem 1.9. Let $\rho: H \rightarrow G L(V)$ be a representation of a compact Lie group $H$ and $X, Y: V \rightarrow$ $V$ two $H$-invariant vector fields that are isomorphic, i.e., differ by a vector field $\psi_{V}$ induced by an equivariant function $\psi: V \rightarrow \mathfrak{h}$. Suppose $X(0)=0$. Then $Y(0)=0$ and

$$
D X(0)=D Y(0)+\delta \rho(\psi(0)) .
$$

Here $\delta \rho: \mathfrak{h} \rightarrow \mathfrak{g l}(V)$ is the corresponding representation of the Lie algebra $\mathfrak{h}$ of $H$.

Together Theorems 1.7 and 1.9 quantity the ambiguity of linearization at relative equilibria:

Theorem 1.10. Let $X, X^{S_{1}}, X^{S_{1}}$ etc. be as in Theorem 1.7. Then

$$
D\left(X^{S_{2}}\right)\left(x_{2}\right)-T \varphi_{x_{1}} D\left(X^{S_{1}}\right)\left(x_{1}\right) \in \delta \rho\left(\psi\left(\mathfrak{h}_{2}^{H_{2}}\right)\right)
$$

where $\rho: H_{2} \rightarrow G L\left(T_{x_{2}} S_{2}\right)$ is the slice representation and $\mathfrak{h}_{2}^{H_{2}}$ is the space of vectors in the Lie algebra of $\mathrm{H}_{2}$ fixed by the adjoint representation.

In particular the linear map $T \varphi_{x_{1}}: T_{x_{1}} S_{x_{1}} \rightarrow T_{x_{2}} S_{x_{2}}$ sends the eigenvectors of the linearization $D\left(X^{S_{1}}\right)\left(x_{1}\right)$ to the eigenvectors of $D\left(X^{S_{2}}\right)\left(x_{2}\right)$, and the real parts of the corresponding eigenvalues are the same.

Remark 1.12. Theorem 1.10 is sharper than [5, Lemma 8.5.2]. For example, if the space $\rho\left(\psi\left(\mathfrak{h}_{2}^{H_{2}}\right)\right)$ is zero then the $T \varphi_{x_{1}}$ defines an isomorphism between the linearizations $D\left(X^{S_{2}}\right)\left(x_{2}\right)$ and $D\left(X^{S_{1}}\right)\left(x_{1}\right)$. See Example 1.13 below.

Example 1.13. Consider a particle of mass $m$ in 3 -space subject to a central force field $F$. Then the phase space of the system is $T \mathbb{R}^{3} \simeq \mathbb{R}^{3} \times \mathbb{R}^{3}$, the equations of motion are of the form

$$
\left\{\begin{aligned}
\dot{q} & =v \\
m \dot{v} & =F(q, v)
\end{aligned}\right.
$$

and the force $F: \mathbb{R}^{3} \times \mathbb{R}^{3} \rightarrow \mathbb{R}^{3}$ is $O(3)$-invariant:

$$
F(A q, A v)=F(q, v) \quad \text { for all } q, v \in \mathbb{R}^{3} \text {, all } A \in O(3) \text {. }
$$

The action of $O(3)$ on $\mathbb{R}^{3} \times \mathbb{R}^{3}$ has 3 orbit types. In more detail, $(0,0)$ is the only fixed point. For $(q, v) \in \mathbb{R}^{3} \times \mathbb{R}^{3}$ with $q$ and $v$ linearly independent the stabilizer is isomorphic to $\{ \pm 1\}=$ "O(1)". If $q, v$ are two linearly dependent vectors with $(q, v) \neq(0,0)$, then the stabilizer of $(q, v)$ is isomorphic to $O(2)$. In all cases the space $\mathfrak{h}^{H}$ of fixed points in the Lie algebra $\mathfrak{h}$ of a stabilizer $H$ is zero. Consequently the linearization of the vector field

$$
X=\sum_{i=1}^{3}\left(v_{i} \frac{\partial}{\partial q_{i}}+\frac{1}{m} F_{i}(q, v) \frac{\partial}{\partial v_{i}}\right)
$$

at any relative equilibrium is well-defined - it doesn't matter which slice through the relative equilibrium we pick, and it doesn't matter which projection of the vector field $X$ onto the slice we choose in order to compute the linearization. 
Remark 1.14. Theorem 1.7 holds for families of vector fields: if $\left\{X_{\lambda}\right\}_{\lambda \in \Lambda}$ is a family of invariant vector fields then the vector fields $\varphi_{*}\left(X_{\lambda}{ }^{S_{1}}\right)$ and $X_{\lambda}{ }^{S_{2}}$ are isomorphic with the isomorphism depending smoothly on $\lambda$.

We next address the issue of genericity. For the purposes of this paper a set is residual if it is the intersection of countably many open dense sets. A vector field on a manifold $M$ is generic if it belongs to a residual subset of $\Gamma(T M)$, Now suppose once again that we have a proper action of a Lie group $G$ on a manifold $M, H$ is the stabilizer of a point $x \in M$ and $S$ is a slice through $x$. We then have a canonical injective linear map

$$
\Gamma(T S)^{H} \hookrightarrow \Gamma(T(G \cdot S))^{G}
$$

from the space of $H$-invariant vector field on the slice $S$ to the space of $G$-invariant vector fields on the tube $G \cdot S$. It is clear that the image has infinite codimension. So the notion of being generic in these two spaces clearly don't correspond. A solution to the problem is provided by:

Theorem 1.16. The map (1.15) induces an invertible linear map

$$
\Gamma(T S)^{H} / C^{\infty}(S, \mathfrak{h})^{H} \rightarrow \Gamma(T(G \cdot S))^{G} / C^{\infty}(G \cdot S, \mathfrak{g})^{G}
$$

from the space of isomorphism classes of invariant vector fields on the slice to the space of isomorphism classes of invariant vector fields on the tube. Moreover if the spaces $\Gamma(T S)^{H}, C^{\infty}(S, \mathfrak{h})^{H}, \Gamma(T(G \cdot S))^{G}$ and $C^{\infty}(G \cdot S, \mathfrak{g})^{G}$ are given Whitney topologies, then the map (1.17) is a homeomorphism. In other words it is an isomorphism of semi-topological spaces.

We now make a reasonable assumption that if an invariant vector field is generic than every vector field isomorphic to it is generic as well. This is equivalent to requiring that the set of generic vector fields $\Gamma(T(G \cdot S))^{G}$ lie in the preimage of a residual set in the quotient $\Gamma(T(G \cdot S))^{G} / C^{\infty}(G \cdot S, \mathfrak{g})^{G}$. With this assumption Theorem 1.16 tells us that a vector field $X \in \Gamma(T S)^{H}$ is generic if and only if its image under the map (1.15) is generic. Thus Theorem 1.16 should be useful in equivariant bifurcation theory. We plan to address this elsewhere.

Theorem 1.16 is a decategorification of a stronger result, Theorem 4.3, which proves that the map (1.15) is part of a chain homotopy equivalence of 2-term chain complexes of semi-topological vector spaces.

The paper is organized as follows. In section 2 we prove Theorem 1.6. In section 3 we prove Theorem 1.7, Theorem 1.9 and Theorem 1.10. In section 4 we prove Theorem 4.3. In the last section, section 5, we explain where the notion of isomorphic vector fields comes from and describe the connections with groupoids and stacks.

\section{ISOMORPHIC VECTOR FIELDS AND THEIR FLOWS}

The goal of this section is to prove Theorem 1.6. Throughout the section a Lie group $G$ with Lie algebra $\mathfrak{g}$ acts properly on a manifold $M$. We do not assume that $G$ is compact. For a point $m \in M$ we have the evaluation map

$$
e v_{m}: G \rightarrow M, \quad e v_{m}(a)=a \cdot m \quad \text { for all } a \in G .
$$

Dually, for any $a \in G$ we have a diffeomorphism

$$
a_{M}: M \rightarrow M, \quad a_{M}(m)=a \cdot m \quad \text { for all } m \in M .
$$

Recall that every $\xi \in \mathfrak{g}$ we have the induced vector field $\xi_{M} \in \Gamma(T M)$ defined by

$$
\xi_{M}(m)=\left.\frac{d}{d t}\right|_{0} \exp (t \xi) \cdot m
$$

Note that by the chain rule

$$
\left.\frac{d}{d t}\right|_{0} \exp (t \xi) \cdot m=\left.\frac{d}{d t}\right|_{0}\left(e v_{m}(\exp t \xi)\right)=T\left(e v_{m}\right)_{0} \xi .
$$


Thus

$$
\xi_{M}(m)=T\left(e v_{m}\right)_{0} \xi
$$

Remark 2.3. We trust that the reader will have no difficulty distinguishing between the induced vector fields $\xi_{M}(2.1)$ and $\psi_{M}(1.4)$.

Proof of Theorem 1.6. Recall that we have a manifold $M$ with an action of a Lie group $G, X, Y \in$ $\Gamma(T M)^{G}$ are two $G$-invariant vector fields and $\psi: M \rightarrow \mathfrak{g}$ is a $G$-equivariant function with

$$
X=Y+\psi_{M} \text {. }
$$

Fix a point $z \in M$. Then

$$
\gamma(t):=\Phi_{t}^{Y}(z)
$$

is an integral curve of $Y$ through $z$. We'd like to prove the existence of a curve $g(t)$ in $G$ which depends smoothly on $z$ so that

$$
\sigma(t):=g(t) \cdot \gamma(t)
$$

is an integral curve of the vector field $X$ through $z$. It is well-known (see, for example, [3, Proposition 1.13.4]) that for any smooth curve $\tau: I \rightarrow \mathfrak{g}$ in the Lie algebra $\mathfrak{g}$ there is a unique smooth curve $g: I \rightarrow G$ defined on the same interval $I$ so that $g(0)=e$ and $g(t)$ solves the ODE

$$
\dot{g}(t)=T R_{g(t)} \tau(t) \text {. }
$$

Here and elsewhere in the paper $R_{a}: G \rightarrow G$ denotes the right multiplication by $a \in G$. In our case we set

$$
\tau(t)=\psi(\gamma(t))=\psi\left(\Phi_{t}^{Y}(z)\right)
$$

We now check that the solutions $g(t)$ of the ODE

$$
\dot{g}(t)=T R_{g(t)} \psi\left(\Phi_{t}^{Y}(z)\right), \quad g(0)=e,
$$

which depend smoothly on $z$ define the desired time-dependent family of maps $\left\{F_{t}: M \rightarrow G\right\}$. For that it is enough to check that

$$
\sigma(t):=g(t) \cdot \gamma(t)
$$

is the integral curve of $X$ through $z$. By the chain rule

$$
\left.\frac{d}{d t}\right|_{0}(g(t) \cdot \gamma(t))=T\left(g(t)_{M}\right)_{\gamma(t)} \dot{\gamma}(t)+T\left(e v_{\gamma(t)}\right)_{g(t)} \dot{g}(t) .
$$

By definition of $\gamma(t)$,

$$
T\left(g(t)_{M}\right)_{\gamma(t)} \dot{\gamma}(t)=T\left(g(t)_{M}\right)_{\gamma(t)} Y(\gamma(t))=Y(g(t) \cdot \gamma(t))=Y(\sigma(t)),
$$

where the next to last equality holds by $G$-invariance of the vector field $Y$. On the other hand,

$$
\begin{aligned}
T\left(e v_{\gamma(t)}\right)_{g(t)} \dot{g}(t) & =T\left(e v_{\gamma(t)}\right)_{g(t)} T R_{g(t)} \psi(\gamma(t)) \quad \text { by definition of } g(t) \\
& =\left.\frac{d}{d s}\right|_{0}(g(t) \exp (s \psi(\gamma(t)))) \cdot \gamma(t) \\
& =\left.\frac{d}{d s}\right|_{0}\left(g(t) \exp (s \psi(\gamma(t))) g(t)^{-1}\right) \cdot g(t) \cdot \gamma(t) \\
& =\left.\frac{d}{d s}\right|_{0}(\exp (s A d(g(t)) \psi(\gamma(t))) \cdot g(t) \cdot \gamma(t) \\
& =\left.\frac{d}{d s}\right|_{0}(\exp (s \psi(g(t) \cdot \gamma(t)))) \cdot(g(t) \cdot \gamma(t)) \quad \text { by equivariance of } \psi \\
& =\psi_{M}(g(t) \cdot \gamma(t))=\psi_{M}(\sigma(t)) .
\end{aligned}
$$

Therefore

$$
\left.\frac{d}{d t}\right|_{0} \sigma(t)=Y(\sigma(t))+\psi_{M}(\sigma(t))=X(\sigma(t))
$$


and we are done.

Remark 2.6. Something about the proof of the theorem may look vaguely familiar to some readers. Indeed it looks very much like the reconstruction argument in symplectic reduction theory. See for instance the discussion at the bottom of p. 304 in [1]. From the point of view of Theorem 1.6 the reconstruction argument says that the horizontal lift of the reduced vector field with respect to some connection and the original Hamiltonian vector field are isomorphic.

Definition 2.7. Since the orbit $G \cdot x$ through a relative equilibrium $x$ of a vector field $X$ is preserved by the flow of $X$ it makes sense to define the relative equilibrium $x$ to be hyperbolic if the manifold $G \cdot x$ is normally hyperbolic for the flow of $X$.

Corollary 2.8. Suppose $X, Y \in \Gamma(T M)^{G}$ are two isomorphic invariant vector fields. Then their flows induce the same flow on the space of orbits $M / G$.

Consequently isomorphic vector fields have the same relative equilibria and relative periodic orbits. Moreover if $x$ is a hyperbolic relative equilibrium for $X$ it is hyperbolic for any vector field $Y$ isomorphic to $X$.

Remark 2.9. The first part of the corollary has a converse if the action of $G$ on $M$ is free and proper: if $X$ and $Y$ are two $G$-invariant vector fields inducing the same flow on the orbit space $B:=M / G$ then $X$ and $Y$ are isomorphic.

The argument proceeds as follows. Since the action of $G$ is free and proper, the orbit space $B:=M / G$ is a manifold and the orbit map $\pi: M \rightarrow B$ makes $M$ into a principal $G$-bundle over $M$. Then two $G$-invariant vector fields $X$ and $Y$ induce the same flow on $B$ if and only if they are $\pi$-related to the same vector field on $B$ if and only if $T \pi(X-Y)=0$ if and only if for every $m \in M$ there is a vector $\psi(m) \in \mathfrak{g}$ so that $X(m)-Y(m)=(\psi(m))_{M}(m)$. It is also easy to see that the function $\psi: M \rightarrow \mathfrak{g}$ is smooth:

$$
\psi(m)=\alpha_{m}(X(m)-Y(m)),
$$

where $\alpha \in \Omega^{1}(M, \mathfrak{g})^{G}$ is a connection 1-form (any choice of $\alpha$ will do). Thus $X$ and $Y$ are isomorphic with the isomorphism provided by the $G$-equivariant map $\psi$ defined above.

\section{LOCAL NORMAL FORM FOR AN INVARIANT VECTOR FIELD}

Once again let $S$ denote a slice through a point $x \in M$ for a proper action of a Lie group $G$ on a manifold $M$ and $H$ denote the stabilizer of $x$. Then

$$
G \cdot S:=\{g \cdot y \mid g \in G, y \in S\}
$$

is an open $G$-invariant neighborhood of the orbit $G \cdot x$, which is often called a tube. We have a $G$-equivariant diffeomorphism $\varphi$ from the associated bundle

$$
G \times{ }^{H} S:=(G \times S) / H
$$

to the tube $G \cdot S$ making the diagram

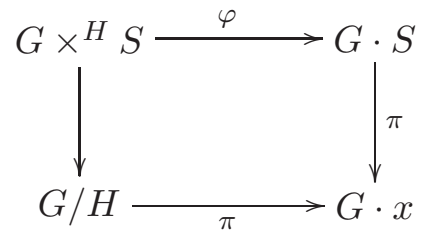

commute. Here the left vertical map is

$$
[g, s] \mapsto g H
$$

the bottom horizontal map is

$$
g H \mapsto g \cdot x
$$

and the right vertical map $\pi: G \cdot S \rightarrow G \cdot x$ is given by

$$
\pi(g \cdot y)=g \cdot x
$$


it is well-defined.

Remark 3.1. The projection $\pi: G \cdot S \rightarrow G \cdot x$ very much depends on the choice of the slice $S$ : the fiber of $\pi$ above $g \cdot x \in G \cdot x$ is the submanifold $g \cdot S$, which is a slice through $g \cdot x$. A choice of a different slice $S^{\prime}$ through $x$ with $G \cdot S^{\prime}=G \cdot S$ defines a different submersion $\pi^{\prime}: G \cdot S^{\prime} \rightarrow G \cdot x$, even though it is given by a seemingly identical formula:

$$
\pi^{\prime}\left(g \cdot y^{\prime}\right)=g \cdot x
$$

for all $g \in G, y^{\prime} \in S^{\prime}$. In particular $\pi$ and $\pi^{\prime}$ have different fibers.

Lemma 3.2. Let $M$ be a manifold with an action of a Lie group $G, H$ the stabilizer of a point $x \in M$ and $S$ a slice through $x$ for the action of $G$. A choice of an $H$-equivariant splitting

$$
\mathfrak{g}=\mathfrak{h} \oplus \mathfrak{m}
$$

of the Lie algebra $\mathfrak{g}$ of $G$ into the Lie algebra $\mathfrak{h}$ of $H$ and a complement $\mathfrak{m}$ gives rise to an isomorphism of vector spaces

$$
\Gamma(T(G \cdot S))^{G} \rightarrow \Gamma(T S)^{H} \oplus C^{\infty}(S, \mathfrak{m})^{H} .
$$

Proof. It will be convenient for notational purposes to assume that $G \cdot S=M$. Then, since $S$ is a global slice, any $G$-invariant vector field $X$ on $M$ is uniquely determined by its restriction to $S$. Thus the restriction map

$$
\left.\Gamma(T M)^{G} \rightarrow \Gamma(T M)^{G}\right|_{S},\left.\quad X \rightarrow X\right|_{S}
$$

is an isomorphism of vector spaces.

The splitting (3.3) defines a $G$-invariant connection on the principal $H$-bundle $G \rightarrow G \cdot x$ and consequently a $G$-invariant connection on the associated bundle $M \simeq G \times{ }^{H} S \rightarrow G \cdot x$. Hence any $G$-invariant vector field $X$ on $M$ can be uniquely written as a sum

$$
X=X^{\mathrm{v}}+X^{\mathrm{h}}
$$

of two $G$-invariant vector fields with $X^{\mathrm{v}}$ being tangent to the fibers of $\pi: M \rightarrow G \cdot x$ and $X^{\mathrm{h}}$ being tangent to the horizontal distribution $\mathcal{H} \subset T M$ defined by the splitting (3.3). It follows that the restriction

$$
X^{S}:=\left.X^{\mathrm{v}}\right|_{S}
$$

is tangent to $S$. It is easy to see that $X^{S}$ is $H$-invariant.

The restriction $\left.\mathcal{H}\right|_{S}$ of the horizontal distribution to the slice is trivial: an isomorphism is given by

$$
S \times\left.\mathfrak{m} \rightarrow \mathcal{H}\right|_{S}, \quad(y, \xi) \mapsto \xi_{M}(y) .
$$

It follows that the space of $H$-equivariant sections of $\left.\mathcal{H}\right|_{S} \rightarrow S$ is isomorphic to the space of $H$ equivariant function $C^{\infty}(S, \mathfrak{m})^{H}$ :

$$
\Gamma\left(\left.\mathcal{H}\right|_{S}\right)^{H} \simeq C^{\infty}(S, \mathfrak{m})^{H}
$$

and the result follows.

Lemma 3.2 tells us that a choice of a slice $S$ and of a splitting (3.3) defines a surjective linear map

$$
\mathfrak{p}: \Gamma(T G \cdot S)^{G} \rightarrow C^{\infty}(S, \mathfrak{m})^{H}, \quad X \mapsto \psi_{X}^{S} .
$$

Remark 3.9. The function $\psi_{X}^{S} \in C^{\infty}(S, \mathfrak{m})^{H}$ and the vector field $X^{\mathrm{h}}$ in (3.5) are, of course, directly related. Indeed, define $\left.\Psi^{X} \in C^{\infty}(G \cdot S), \mathfrak{g}\right)^{G}$ by

$$
\Psi_{X}(g \cdot y)=A d(g) \psi_{X}^{S}(y) .
$$

Then

$$
X^{\mathrm{h}}=\left(\Psi_{X}\right)_{M}(m)
$$

for all $m \in G \cdot S$, and the decomposition (3.5) reads:

$$
X(g \cdot y)=T\left(g_{M}\right)_{y} X^{S}(y)+\left(\Psi_{X}\right)_{M}(g \cdot y)
$$

for all $y \in S, g \in G$. 
Lemma 3.11. Let $S, S^{\prime}$ be two different slices through the same point $x$ for a proper action of a Lie group $G$ on a manifold $M$. Let $H$ denote the stabilizer of $x$, as before. There exists an $H$-equivariant $G$-valued function $f$ defined on a neighborhood $U$ of $x$ in $S$ so that

$$
\varphi: U \rightarrow S^{\prime}, \quad \varphi(y)=f(y) \cdot y
$$

is an $H$-equivariant open embedding. Moreover we may assume $f$ takes values in exp $\mathfrak{m}$, where $\mathfrak{g}=\mathfrak{h} \oplus \mathfrak{m}$ is an $H$-equivariant splitting.

Proof. We may assume that $M=G \times{ }^{H} S$ and $S^{\prime}$ is a slice through $[1, x] \in G \times{ }^{H} S$, where $[1, x]$ is the $H$-orbit of $(1, x) \in G \times S$. It is a standard fact that an $H$-invariant splitting $\mathfrak{g}=\mathfrak{m} \oplus \mathfrak{h}$ defines an $H$-equivariant section $s$ of the principal $H$ bundle $G \rightarrow G / H$. Explicitly the section is given by the formula

$$
s(g H)=g
$$

for all $g \in \exp (\mathfrak{m})$ sufficiently close to 1 , say for $g$ in an $H$-invariant neighborhood $\mathcal{O}$ of $1 \in \exp (\mathfrak{m})$.

The section $s$ trivializes the associated bundle $\pi: G \times{ }^{H} S \rightarrow G / H=G \cdot x$. Explicitly the trivialization is the map

$$
\mathcal{O} \times S \rightarrow \pi^{-1}(\mathcal{O} G), \quad(g, y) \mapsto[g, y]=g \cdot[1, y] .
$$

We may assume that $S^{\prime} \subset \mathcal{O} \times S$ and that at $(1, x) \in \mathcal{O} \times S$ we have

$$
T_{(1, x)}(\mathcal{O} \times S)=T_{(1, x)} \mathcal{O} \oplus T_{(1, x)} S^{\prime} .
$$

Consequently the differential of $\left.p r_{2}\right|_{S^{\prime}}: S^{\prime} \rightarrow S$ at $(1, x)$ is an isomorphism, hence a diffeomorphism from a neighborhood $U^{\prime}$ of $(1, x) \in S^{\prime}$ to a neighborhood $U$ of $x$ in $S$. Since $p r_{2}$ is $H$-equivariant, $\left.p r_{2}\right|_{S^{\prime}}$ is $H$-equivariant as well, and we may take $U, U^{\prime}$ to be $H$-invariant. We set

$$
\varphi=\left(\left.p r_{2}\right|_{U^{\prime}}\right)^{-1}: U \rightarrow U^{\prime}
$$

It is the desired map, and it's of the form

$$
\varphi(y)=(f(y), y)
$$

for some $H$-equivariant map $f: U \rightarrow \mathcal{O} \subset \exp (\mathfrak{m})$.

When we identify $G \times{ }^{H} S$ with $G \cdot S$ the map $\varphi$ takes the form

$$
\varphi(y)=f(y) \cdot y,
$$

as desired.

Lemma 3.13. Let $\varphi: S \rightarrow S^{\prime}, \varphi(y)=f(y) \cdot y$ be the equivariant map of Lemma 3.11. Denote the left multiplication by an element $a \in G$ by $L_{a}$. For any $y \in S, v \in T_{y} S$,

$$
T \varphi_{y}(v)=T\left(f(y)_{M}\right)_{y}\left[\left(T L_{f(y)^{-1}}\left(T f_{y}(v)\right)\right)_{M}(\varphi(y))+v\right] .
$$

Remark 3.15. In (3.14) $f(y) \in G, f(y)_{M}: M \rightarrow M$ is the corresponding diffeomorphism, and $T\left(f(y)_{M}\right)_{y}: T_{y} M \rightarrow T_{f(y)} M$ is its differential. Similarly $T f_{y}: T_{y} M \rightarrow T_{f(y)} G$ is the differential of $f$ at $y, T L_{f(y)^{-1}}\left(T f_{y}(v)\right) \in T_{1} G=\mathfrak{g}$, and $\left(T L_{f(y)^{-1}}\left(T f_{y}(v)\right)\right)_{M}(\varphi(y))$ is the value at $\varphi(y)$ of the vector field on $M$ induced by the vector $T L_{f(y)^{-1}}\left(T f_{y}(v)\right) \in \mathfrak{g}$.

Proof of Lemma 3.13. The derivative $T\left(e v_{y}\right)_{g}$ of the evaluation map

$$
e v_{y}: G \rightarrow M, \quad e v_{y}(g)=g \cdot y
$$

at a point $g \in G$ can be computed as follows: For $w \in T_{g} G$ set

$$
z=T L_{g^{-1}} w \in T_{e} G=\mathfrak{g} .
$$

Then $w=T L_{g} z$ and

$$
T\left(e v_{y}\right) w=\left.\frac{d}{d t}\right|_{0} e v_{y}\left(L_{g}(\exp t z)\right)=\left.\frac{d}{d t}\right|_{0} g(\exp t z) y=T\left(g_{M}\right)_{y}\left(T L_{g^{-1}} w\right)_{M}(y) .
$$


Next choose a curve $\gamma: I \rightarrow M$ with $\gamma(0)=y, \dot{\gamma}(0)=v$. Then

$$
\begin{aligned}
T \varphi_{y}(v) & =\left.\frac{d}{d t}\right|_{0} \varphi(\gamma(t))=\left.\frac{d}{d t}\right|_{0}(f(\gamma(t)) \cdot \gamma(t))= \\
& =\left.T\left(e v_{\gamma(0)}\right) \frac{d}{d t}\right|_{0} f(\gamma(t))+T(f(\gamma(0)))_{M}(\dot{\gamma}(0)) \\
& =T\left(f(y)_{M}\right)_{y}\left(\left(T L_{g^{-1}} T f_{y}(v)\right)_{M}(y)+v\right) . \quad \text { by }(3.16)
\end{aligned}
$$

Proof of Theorem 1.\%. We first address the dependence of the projection

$$
\mathfrak{q}: \Gamma(T(G \cdot S))^{G} \rightarrow \Gamma(T S)^{H}
$$

on the choice of the splitting $\mathfrak{g}=\mathfrak{h} \oplus \mathfrak{m}$.

\section{Lemma 3.17. Let}

$$
\mathfrak{g}=\mathfrak{h} \oplus \mathfrak{m}_{1}=\mathfrak{h} \oplus \mathfrak{m}_{2}
$$

be two $H$-equivariant splittings and

$$
\mathfrak{q}_{1}, \mathfrak{q}_{2}: \Gamma(T(G \cdot S))^{G} \rightarrow \Gamma(T S)^{H}
$$

the two corresponding projections. Then for any $X \in \Gamma(T(G \cdot S))^{G}$ the two vector fields

$$
X_{i}:=\mathfrak{q}_{i}(X), \quad i=1,2
$$

are isomorphic in the sense of Definition 1.5: there is map $\varphi \in C^{\infty}(S, \mathfrak{h})^{H}$ with

$$
X_{1}-X_{2}=\varphi_{S} \text {. }
$$

Proof. Since $\mathfrak{m}_{1}, \mathfrak{m}_{2}$ are both complementary to $\mathfrak{h}$ in $\mathfrak{g}$ there exists a linear map

$$
B: \mathfrak{m}_{1} \rightarrow \mathfrak{h}
$$

so that $\mathfrak{m}_{2}$ is the graph of $B$ :

$$
\mathfrak{m}_{2}=\left\{v+B(v) \in \mathfrak{g} \mid v \in \mathfrak{m}_{1}\right\} .
$$

Since the splittings (3.18) are $H$-equivariant, the map $B$ is $H$-invariant. Define $\psi_{i} \in C^{\infty}\left(S, \mathfrak{m}_{i}\right)^{H}$, $(i=1,2)$, by

$$
\psi_{i}(y):=\left(\left.T\left(e v_{x}\right)_{0}\right|_{\mathfrak{m}_{i}}\right)^{-1}\left(T \pi_{y}(X(y))\right.
$$

Then

$$
\psi_{1}(y)+B\left(\psi_{1}(y)\right) \text { is in } \mathfrak{m}_{2} \text { for all } y \in S .
$$

Since $B\left(\psi_{1}(y)\right) \in \mathfrak{h}$, we have

$$
T\left(e v_{x}\right)_{0}\left(B\left(\psi_{1}(y)\right)\right)=0 \quad \text { for all } y \in S
$$

Hence

$$
T\left(e v_{x}\right)_{0}\left(\psi_{1}(y)+B\left(\psi_{1}(y)\right)\right)=T\left(e v_{x}\right)_{0}\left(\psi_{1}(y)\right)=T \pi_{y}(X(y))=T\left(e v_{x}\right)_{0}\left(\psi_{2}(y)\right) .
$$

Since $T\left(\left.\left(e v_{x}\right)_{0}\right|_{\mathfrak{m}_{2}}\right.$ is $1-1$, it follows that

$$
\psi_{1}(y)+B\left(\psi_{1}(y)\right)=\psi_{2}(y) .
$$

We define $\varphi: S \rightarrow \mathfrak{h}$ by

it is $H$-equivariant. Then

$$
\varphi(y)=B\left(\psi_{1}(y)\right)
$$

$$
\psi_{2}-\psi_{1}=\varphi
$$

Moreover

$$
\left(X_{1}-X_{2}\right)(y)=\left(X(y)-\left(\psi_{1}\right)_{M}(y)\right)-\left(X(y)-\left(\psi_{2}\right)_{M}(y)\right)=\left(\psi_{2}-\psi_{1}\right)_{M}(y)=\varphi_{M}(y),
$$

which proves the lemma. 
Next we address the dependence of the projection

$$
\mathfrak{q}: \Gamma(T(G \cdot S))^{G} \rightarrow \Gamma(T S)^{H}
$$

on the point $x$. For any $g \in G$

$$
S^{\prime}:=g_{M}(S) \quad(=g \cdot S)
$$

is a slice through $g \cdot x$, the stabilizer of $g \cdot x$ is $H^{\prime}=g H g^{-1}$, and an $H$-equivariant splitting $\mathfrak{g}=\mathfrak{h} \oplus \mathfrak{m}$ defines an $H^{\prime}$-equivariant splitting

$$
\mathfrak{g}=A d(g) \mathfrak{h} \oplus A d(g) \mathfrak{m}=\mathfrak{h}^{\prime} \oplus A d(g) \mathfrak{m} .
$$

Since $g_{M}: S \rightarrow S^{\prime}$ is an $H-H^{\prime}$-equivariant diffeomorphism, it defines an isomorphism

$$
g_{*}: \Gamma(T S)^{H} \rightarrow \Gamma\left(T S^{\prime}\right)^{H^{\prime}},
$$

given by

$$
\left(g_{*} Y\right)\left(y^{\prime}\right)=T g_{M} Y\left(g^{-1} \cdot y^{\prime}\right), \quad y^{\prime} \in S^{\prime} .
$$

Equation (3.10) now implies that the image $X^{S^{\prime}}$ of $X \in \Gamma(T(G \cdot S))^{G}$ under the projection

$$
\mathfrak{q}^{\prime}: \Gamma(T(G \cdot S))^{G} \rightarrow \Gamma\left(T S^{\prime}\right)^{H^{\prime}}
$$

(which is defined by the slice $S^{\prime}$ and the splitting $\mathfrak{g}=\mathfrak{h}^{\prime} \oplus A d(g) \mathfrak{m}$ ) is exactly $g_{*} X^{S}$. In other words, in this case

$$
X^{S^{\prime}} \text { and } g_{*} X^{S} \text { are equal. }
$$

Therefore, to finish our proof of Theorem 1.7 it is enough to show:

Lemma 3.21. Suppose $S, S^{\prime}$ are two slices through the same point $x$. Let $\varphi: S \rightarrow S^{\prime}, \varphi(y)=f(y) \cdot y$ be the equivariant diffeomorphism of Lemma 3.11. Then for any $G$-invariant vector field $X$ on the original manifold $M$ the vector fields $X^{S^{\prime}}$ and $\varphi_{*} X^{S} \in \Gamma\left(T S^{\prime}\right)^{H}$ are isomorphic: there exists an $H$-equivariant map $\nu: S^{\prime} \rightarrow \mathfrak{h}$ with

$$
X^{S^{\prime}}-\varphi_{*} X^{S}=\nu_{S}
$$

Here $\nu_{S}\left(y^{\prime}\right)=\left.\frac{d}{d t}\right|_{0}\left(\exp t \nu\left(y^{\prime}\right)\right) \cdot y^{\prime}$ and $\varphi_{*} X^{S}\left(y^{\prime}\right)=T \varphi_{\varphi^{-1}\left(y^{\prime}\right)} X^{S}\left(\varphi^{-1}\left(y^{\prime}\right)\right)$ for $y^{\prime} \in S^{\prime}$.

Proof. As before fix an $H$-equivariant splitting $\mathfrak{g}=\mathfrak{h} \oplus \mathfrak{m}$. Then by Remark 3.9 for any point $y$ in the slice $S$ we have

$$
X^{S}(y)=X(y)-\left(\Psi^{S}\right)_{M}(y)
$$

with $X^{S} \in \Gamma(T S)^{H}$ and $\Psi^{S} \in C^{\infty}(G \cdot S, \mathfrak{g})^{G}$. Similarly

$$
X^{S^{\prime}}\left(y^{\prime}\right)=X\left(y^{\prime}\right)-\left(\Psi^{S^{\prime}}\right)_{M}\left(y^{\prime}\right)
$$

for $y^{\prime} \in S^{\prime}, X^{S^{\prime}} \in \Gamma\left(T S^{\prime}\right)^{H}$ and $\Psi^{S^{\prime}} \in C^{\infty}(G \cdot S, \mathfrak{g})^{G}$. By Lemma 3.13, for any $y \in S$,

$$
T \varphi_{y}\left(X^{S}(y)\right)=T\left(f(y)_{M}\right)_{y}\left(X^{S}(y)+\mu_{M}(y)\right),
$$

where $\mu \in C^{\infty}(G \cdot S, \mathfrak{g})^{G}$ is defined by

$$
\mu(y)=T L_{f(y)^{-1}}\left(T f_{y}(X(y))\right)
$$

for $y \in S$ and $\mu(g \cdot y)=A d(g) \mu(y)$ for an arbitrary $g \cdot y \in G \cdot S$. Recall that for any $G$-invariant vector field $Z$ on $G \cdot S$

$$
T\left(g_{M}\right) Z(y)=Z(g \cdot y)
$$

for all $g \in M$ and $y \in S$. Therefore, for $y \in S, y^{\prime}=f(y) \cdot y \in S^{\prime}$,

$$
\begin{aligned}
X^{S^{\prime}}\left(y^{\prime}\right)-\varphi_{*} X^{S}\left(y^{\prime}\right) & =\left(X\left(y^{\prime}\right)-\left(\Psi^{S^{\prime}}\right)_{M}\left(y^{\prime}\right)\right)-T\left(f(y)_{M}\right)_{y}\left(X^{S}(y)+\mu_{M}(y)\right) \\
& =X\left(y^{\prime}\right)-\left(\Psi^{S^{\prime}}\right)_{M}\left(y^{\prime}\right)-T\left(f(y)_{M}\right)_{y}\left(X(y)-\left(\Psi^{S}\right)_{M}(y)\right)-\mu_{M}\left(y^{\prime}\right) \\
& =X\left(y^{\prime}\right)-\left(\Psi^{S^{\prime}}\right)_{M}\left(y^{\prime}\right)-X\left(y^{\prime}\right)+\left(\Psi^{S}\right)_{M}\left(y^{\prime}\right)-\mu_{M}\left(y^{\prime}\right) \\
& =\left(\Psi^{S}-\Psi^{S^{\prime}}-\mu\right)_{M}\left(y^{\prime}\right) .
\end{aligned}
$$


Now define $\nu\left(y^{\prime}\right)=\left(\Psi^{S}-\Psi^{S^{\prime}}-\mu\right)\left(y^{\prime}\right)$ for all $y^{\prime} \in S^{\prime}$. Since at points of the slice $S^{\prime}$ the induced vector field $\nu_{M}$ is tangent to $S^{\prime}$, the function $\nu$ takes values in $\mathfrak{h}$.

This concludes our proof of Theorem 1.7.

We now turn out attention to Theorem 1.9.

Proof of Theorem 1.9. Since $X-Y=\psi_{V}, D X(0)-D Y(0)=D\left(\psi_{V}\right)(0)$. Now, for any $v \in V$,

$$
\begin{aligned}
D\left(\psi_{V}\right)(0) v & =\left.\frac{\partial}{\partial s}\right|_{s=0} \psi_{V}(s v)=\left.\frac{\partial^{2}}{\partial s \partial t}\right|_{(0,0)} \rho\left(\exp t \psi_{V}(s v)\right)(s v) \\
& =\left.\frac{\partial^{2}}{\partial s \partial t}\right|_{(0,0)} e^{t \delta \rho\left(\psi_{V}(s v)\right)}(s v)=\left.\frac{\partial}{\partial s}\right|_{s=0} \delta \rho(\psi(s v))(s v) \\
& =\left(\delta \rho\left(\left.\psi(s v)\right|_{s=0}\right)\left(\left.\frac{\partial}{\partial s}\right|_{s=0}(s v)\right)+\left(\left.\frac{\partial}{\partial s}\right|_{s=0}\right)\left(\delta \rho\left(\left.\psi(s v)(s v)\right|_{s=0}\right)\right.\right. \\
& =\delta \rho(\psi(0)) v .
\end{aligned}
$$

We finish the section with a proof of Theorem 1.10

Proof of Theorem 1.10. We may assume that $S_{2}=T_{x_{2}} S_{2}$ and $x_{2}=0 \in T_{x_{2}} S_{2}$ and the action of $H$ on $S_{2}$ is given by the slice representation $\rho: H \rightarrow T_{x_{2}} S_{2}$. By Theorem 1.7 there is an $H$-equivariant map $\psi: S_{2} \rightarrow \mathfrak{h}_{2}$ so that

$$
X^{S_{2}}-\varphi_{*} X^{S_{1}}=\psi_{S_{2}}
$$

By Theorem 1.9

$$
D X^{S_{2}}(0)-D\left(\varphi_{*} X^{S_{1}}\right)(0)=\delta \rho(\psi(0)) .
$$

Since $\psi: T_{x_{2}} S_{2} \rightarrow \mathfrak{h}_{2}$ is $H_{2}$-equivariant and $0 \in T_{x_{2}} S_{2}$ is fixed by the action $H_{2}, \psi(0)$ is fixed by $H_{2}$ as well, i.e., $\left.\xi:=\psi(0) \in \mathfrak{h}_{2}^{H_{2}}\right)$ ). Therefore (1.11) holds. Since $\varphi_{*} X^{S_{1}}$ is an $H_{2}$ invariant vector field, its differential $D\left(\varphi_{*} X^{S_{1}}\right)(0)$ is an $H_{2}$-equivariant linear map from $T_{x_{2}} S_{2}$ to $T_{x_{2}} S_{2}$. Hence $D\left(\varphi_{*} X^{S_{1}}\right)(0)$ and $\delta \rho(\xi)$ commute. Consequently they have the same eigenvalues. Since $D X^{S_{2}}(0)=D\left(\varphi_{*} X^{S_{1}}\right)(0)+\delta \rho(\xi)$, the maps $D X^{S_{2}}\left(x_{2}\right)=D X^{S_{2}}(0)$ and $D\left(\varphi_{*} X^{S_{1}}\right)(0)=T \varphi_{x_{1}} D\left(X^{S_{1}}\right)\left(x_{1}\right)$ have the same eigenvectors as well. Moreover, the eigenvalues of $D X^{S_{2}}(0)$ are sums of the corresponding eigenvalues of $D\left(\varphi_{*} X^{S_{1}}\right)(0)$ and $\delta \rho(\xi)$. Since $H$ is compact the eigenvalues of $\delta \rho(\xi)$ are purely imaginary for all $\xi \in \mathfrak{h}$. Hence the real parts of the eigenvalues of $D X^{S_{2}}(0)$ and $D\left(\varphi_{*} X^{S_{1}}\right)(0)$ are the same.

\section{Genericity in the space of invariant vector fields}

The goal of this section is to prove that the map (1.15) is part of a chain homotopy equivalence of 2 -term chain complexes of semi-topological vector spaces, Theorem 4.3. Theorem 1.16 follows readily from this result. We start by recalling a fact about Whitney $C^{\infty}$ topologies.

Proposition 4.1 ([7, Proposition 3.5, p. 46]). Let $M, N, Q$ be three manifolds and $f \in C^{\infty}(N, Q)$. Then the map $f_{*}: C^{\infty}(M, N) \rightarrow C^{\infty}(M, Q)$ given by $f_{*}(\varphi):=f \circ \varphi$ is continuous in the Whitney $C^{\infty}$ topology.

We apply it as follows.

Proposition 4.2. Let $M$ be a manifold with an action of a Lie group $G$. The map

$$
\partial: C^{\infty}(M, \mathfrak{g})^{G} \rightarrow \Gamma(T M)^{G}, \quad \partial(\psi):=\psi_{M}
$$

is continuous in the Whitney $C^{\infty}$ topology (q.v. (1.4)). In other words $\partial: C^{\infty}(M, \mathfrak{g})^{G} \rightarrow \Gamma(T M)^{G}$ is a 2-term chain complex of semi-topological vector spaces. 
Proof. We argue that the map $\partial$ is continuous. The action of the Lie group $G$ on the manifold $M$ defines a smooth map $(\cdot)_{M}$ from the trivial vector bundle $M \times \mathfrak{g} \rightarrow M$ to the tangent bundle $T M$. The map is defined by

$$
(m, X) \mapsto X_{M}(m):=\left.\frac{d}{d t}\right|_{0} \exp (t X) \cdot m .
$$

Hence by Proposition 4.1 the map

$$
C^{\infty}(M, \mathfrak{g}) \rightarrow \Gamma(T M), \quad \psi \mapsto \psi_{M}=(\cdot)_{M} \circ \psi
$$

is continuous. Consequently its restriction to equivariant functions

$$
(\cdot)_{M}: C^{\infty}(M, \mathfrak{g})^{G} \rightarrow \Gamma(T M)^{G}
$$

is continuous as well.

Theorem 4.3. Let $M$ be a manifold with a proper action of a Lie group $G$ and $S \subset M$ a global slice through $x \in M$ for the action of $G$ so that $M=G \cdot S \simeq G \times{ }^{H} S$, where $H$ is the stabilizer of $S$. Consider the map

$$
K_{\bullet}:\left(C^{\infty}(S, \mathfrak{h})^{H} \stackrel{\partial}{\longrightarrow} \Gamma(T S)^{H}\right) \rightarrow\left(C^{\infty}(G \cdot S, \mathfrak{g})^{G} \stackrel{\partial}{\longrightarrow} \Gamma(T(G \cdot S))^{G}\right)
$$

defined on $C^{\infty}(S, \mathfrak{h})^{H}$ by

$$
K_{1}(\psi)(g \cdot y):=A d(g) \psi(y) \quad \text { for all } g \in G, y \in S
$$

and on $\Gamma(T S)^{H}$ by

$$
K_{0}(Y)(g \cdot y)=T\left(g_{M}\right)_{y} Y(y) \quad \text { for all } g \in G, y \in S .
$$

Then $K_{\bullet}$, which is a map of 2-term chain complexes of semi-topological spaces, is (part of) a chain homotopy equivalence.

Proof. Since

$$
(K(\psi))_{M}(g \cdot y)=T\left(g_{M}\right)_{y} \psi_{S}(y),
$$

$K$ is a map of chain complexes. We have two isomorphisms of semi-topological vector spaces

$$
C^{\infty}(G \cdot S, \mathfrak{g})^{G} \rightarrow C^{\infty}(S, \mathfrak{g})^{H} \quad \text { and } \quad \Gamma(T(G \cdot S))^{G} \rightarrow \Gamma\left(\left.T(G \cdot S)\right|_{S}\right)^{H}
$$

which are both given by restrictions. A choice of an $H$-equivariant splitting $\mathfrak{g}=\mathfrak{h} \oplus \mathfrak{m}$ of the Lie algebra $\mathfrak{g}$ defines two more isomorphisms:

$$
C^{\infty}(S, \mathfrak{g})^{H} \simeq C^{\infty}(S, \mathfrak{h})^{H} \oplus C^{\infty}(S, \mathfrak{m})^{H} \quad \text { and } \quad \Gamma\left(\left.T(G \cdot S)\right|_{S}\right)^{H} \simeq \Gamma(T S)^{H} \oplus C^{\infty}(S, \mathfrak{m})^{H} .
$$

Next observe that the diagram

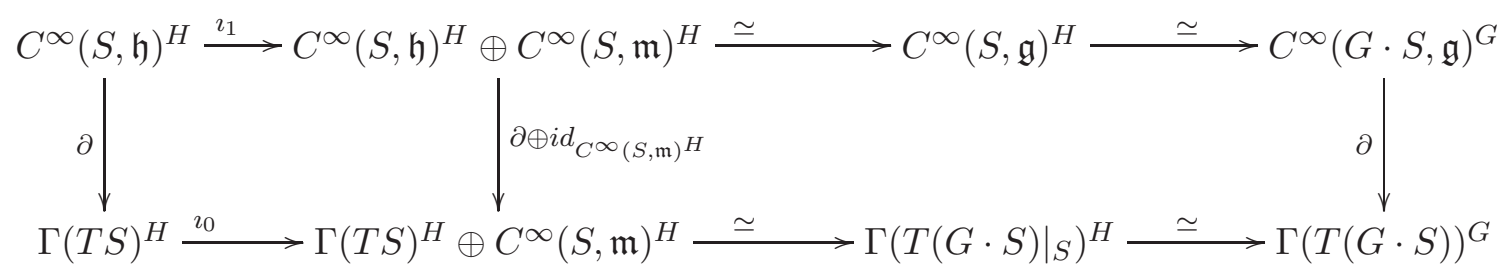

commutes, and that the composites of the horizontal arrows are $K_{1}$ and $K_{0}$, respectively. The projections

$$
p_{1}: C^{\infty}(S, \mathfrak{h})^{H} \oplus C^{\infty}(S, \mathfrak{m})^{H} \rightarrow C^{\infty}(S, \mathfrak{h})^{H} \quad \text { and } \quad p_{0}: \Gamma(T S)^{H} \oplus C^{\infty}(S, \mathfrak{m})^{H} \rightarrow \Gamma(T S)^{H}
$$

are continuous and define a chain homotopy inverse to the chain map $\imath_{\bullet}$. The result follows. 


\section{Relation with the work of Hepworth and Baez-Crans}

A 2-term chain complex $V_{1} \stackrel{\partial}{\rightarrow} V_{0}$ of semi-topological vector spaces defines an action of the abelian topological group $V_{1}$ on $V_{0}$ by

$$
v_{1} * v_{0}:=\partial\left(v_{1}\right)+v_{0} .
$$

The action is continuous since + and $\partial$ are continuous. Hence the corresponding action groupoid

$$
V_{1} \times V_{0} \rightrightarrows V_{0}
$$

is a semi-topological linear groupoid, that is, a groupoid internal to the category of semi-topological vector spaces. The collection of all 2-term chain complexes of semi-topological vector spaces form a 2-category st2term: the 1-morphisms are (continuous) chain maps and 2-morphisms are (continuous) chain homotopies. Semi-topological linear groupoids also form a 2-category; call it stLinGpds: the 1morphisms are (linear continuous) functors and 2-morphisms are natural transformations. The map that assigns to a 2-term chain complex the corresponding action groupoid extends to a 2 -functor

$$
\mathcal{S}_{s t}: \text { st2term } \rightarrow \text { stLinGpds. }
$$

Definition 5.1. Let $M$ be a manifold with an action of a Lie group $G$. The groupoid of invariant vector fields $\mathbb{X}(G \times M \rightrightarrows M)$ is the semi-topological linear groupoid corresponding to the 2-term chain complex:

$$
\partial: C^{\infty}(M, \mathfrak{g})^{G} \rightarrow \Gamma(T M)^{G}, \quad \partial(\psi):=\psi_{M}
$$

of semi-topological vector spaces (q.v. (1.3)).

If we forget about topology and continuity, we get the 2-categories 2term of 2-term chain complexes of vector spaces and $2 \mathrm{Vect}$ of groupoids internal to the category of vector spaces. The 2-category $2 \mathrm{Vect}$ is more commonly known as the 2-category of Baez-Crans 2-vector spaces [2]. Just as in the case of semi-topological vector spaces there is a 2 -functor

$$
\mathcal{S}: 2 \text { term } \rightarrow 2 \text { Vect, }
$$

which assigns to a 2-term chain complex the corresponding action groupoid etc. Baez and Crans proved (op. cit.) that $\mathcal{S}$ is an equivalence of 2-categories. We note that results of this sort had been known before [6], but no detailed accounts were written down. We also note that the analogous statement for $\mathcal{S}_{s t}:$ st2term $\rightarrow$ stLinGpds is unlikely to be true. The problem is that short exact sequences of semi-topological vector spaces need not split. One expects that the problem is fixable at a price one should enlarge the 2-category st2term to a bicategory whose 1-morphisms are analogues of Noohi's butterflies [10].

If once again we forget topology amd assume that the group $G$ is compact, then the groupoid $\mathbb{X}(G \times M \rightrightarrows M)$ can be rigorously interpreted as the groupoid of vector fields on the quotient stack $[M / G]([4$, Proposition 6.1]). Here are the details.

Traditionally given a proper action of a Lie group $G$ on a manifold $M$ one thinks of a quotient $M / G$ as a topological space with some additional structure. For instance one proves that $M / G$ is a stratified space and that it is "smooth" in an appropriate sense. There is also another notion of a quotient that has its origins in the works of Grothendieck, Deligne, Mumford, Artin and their collaborators - this is the notion of a stack quotient $[M / G]$. Stack quotients are instances of geometric stacks and consequently come with atlases. A choice of an atlas for a stack defines a Lie groupoid that "represents" the stack. Two different choices of atlases give rise to Morita equivalent Lie groupoids. It turns out that the notion of a vector field on a stack does make sense, but instead of forming a vector space the collection of vector fields on a given stack $S$ forms a category $\mathcal{X}(S)$. It was shown by Hepworth [4] that if a stack $S$ is represented by a Lie groupoid $\mathscr{G}$ then the category of vector fields on $S$ is equivalent to the category $\mathscr{X}_{\text {mult }}(\mathscr{G})$ of the so called multiplicative vector fields on $\mathscr{G}$.

We recall how the category of multiplicative vector fields, which were defined by Mackenzie and Xu M [9], comes about. Given a groupoid $\mathscr{G}$ there is a tangent groupoid $T \mathscr{G}$ with a canonical functor $\pi: T \mathscr{G} \rightarrow \mathscr{G}$. A multiplicative vector field $X$ on a groupoid $\mathscr{G}$ is a section of $\pi: T \mathscr{G} \rightarrow \mathscr{G}$. In particular it is a functor. Since multiplicative vector fields are functors, it make sense to talk about natural 
transformations between them. Since vector fields take values in groupoids the natural transformations are natural isomorphisms. Thus multiplicative vector fields on a Lie groupoid $\mathscr{G}$ form a category (in fact, a groupoid).

Given an action of a Lie group $G$ on a manifold $M$, we have an action Lie groupoid $G \times M \rightrightarrows M$. An invariant vector field $X: M \rightarrow T M$ extends canonically to a multiplicative vector field

$$
(0, X): G \times M \rightarrow T G \times T M .
$$

Consequently the groupoid $\mathbb{X}(G \times M \rightrightarrows M)$ is contained in the groupoid $\mathscr{X}_{\text {mult }}(G \times M \rightrightarrows M)$ of multiplicative vector fields. Hepworth shows that if $G$ is compact, then inclusion is a fully faithful and essentially surjective functor (if $G$ is not compact this need not be the case). Hence the groupoids $\mathbb{X}(G \times M \rightrightarrows M)$ and $\mathscr{X}_{\text {mult }}(G \times M \rightrightarrows M)$ are equivalent. As was mentioned above the groupoid of multiplicative vector fields $\mathscr{X}_{\text {mult }}(\mathscr{G})$ on a groupoid $\mathscr{G}$, in turn, is equivalent to the groupoid of vector fields $\mathcal{X}(B \mathscr{G})$ on the stack $B \mathscr{G}$ of principal $\mathscr{G}$ bundles. Thus if two Lie groupoids $\mathscr{G}$ and $\mathscr{H}$ are Morita equivalent, then the stacks $B \mathscr{G}$ and $B \mathscr{H}$ are isomorphic and consequently the groupoids of multiplicative vector fields $\mathscr{X}_{\text {mult }}(\mathscr{G})$ and $\mathscr{X}_{\text {mult }}(\mathscr{H})$ are equivalent as well.

On a manifold a vector field integrates to flow, which is, more or less, a one-parameter family of diffeomorphisms. Analogously on a Lie groupoid a multiplicative vector field integrates to a oneparameter family of functors [9] (this is imprecise; a precise statement involves double groupoids). An isomorphism between two multiplicative vector fields should integrate to a one-parameter family of natural isomorphisms between these functors. This is what we proved in Section 2 in the special case of invariant vector fields.

If $S$ is a slice for the action of a group $G$ on a manifold $M$ and $G \cdot S \simeq G \times{ }^{H} S$ is the corresponding tube, then the action groupoids $G \times G \cdot S \rightrightarrows G \cdot S$ and $H \times S \rightrightarrows S$ are Morita equivalent. Consequently the groupoids $\mathbb{X}(G \times G \cdot S \rightrightarrows G \cdot S)$ and $\mathbb{X}(H \times S \rightrightarrows S)$ of invariant vector fields are Morita equivalent (at least when $G$ is compact). This follows from the chain of equivalences of groupoids

$$
\begin{aligned}
\mathbb{X}(H \times S & \rightrightarrows S) \simeq \mathscr{X}_{\text {mult }}(H \times S \rightrightarrows S) \simeq \mathcal{X}([S / H]) \simeq \mathcal{X}([G \cdot S / G]) \\
& \simeq \mathscr{X}_{\text {mult }}(G \times G \cdot S \rightrightarrows G \cdot S) \simeq \mathbb{X}(G \times G \cdot S \rightrightarrows G \cdot S) .
\end{aligned}
$$

So perhaps it is not so surprising that the natural functor

$$
K: \mathbb{X}(H \times S \rightrightarrows S) \rightarrow \mathbb{X}(G \times G S \rightrightarrows G S)
$$

which corresponds to the map of chain complexes $K_{\bullet}$ (q.v. (4.4)) is an equivalence of categories. I think it $i s$ surprising that $K$ is a strong equivalence of semi-topological linear groupoids, that is, the chain map $K_{\bullet}$ has a homotopy inverse.

\section{REFERENCES}

[1] R. Abraham and J.E. Marsden, Foundations of mechanics, second edition, revised and enlarged. With the assistance of Tudor Ratiu and Richard Cushman. Benjamin/Cummings Publishing Co., Inc., Advanced Book Program, Reading, Mass., 1978.

[2] J. Baez and A. Crans, Higher-dimensional algebra VI: Lie 2-algebras, Theory and Applications of Categories 12 (2004) 492-538, arXiv:math.QA/0307263.

[3] J.J. Duistermaat and J.A.C. Kolk, Lie Groups Springer-Verlag, Berlin, Heidelberg, New York, 1999.

[4] R. Hepworth, Vector fields and flows on differentiable stacks, Theory Appl. Categ. 22 (2009), 542-587.

[5] M. Field, Dynamics and symmetry, ICP Advanced Texts in Mathematics, 3. Imperial College Press, London, 2007. xiv $+478 \mathrm{pp}$.

[6] A. Grothendieck, Categories Cofibrees Additives et Complexe Cotangent Relatif, Lecture Notes in Math. 79, SpringerVerlag, New York, 1968.

[7] M. Golubitsky and V. Guillemin, Stable Mappings and Their Singularities, Springer-Verlag, Berlin, Heidelberg, New York, 1973.

[8] M. Krupa, Bifurcations of relative equilibria, SIAM J. Math. Anal., 21(6), 1990, 1453-1486.

[9] K.C.H. Mackenzie and P. Xu, Classical lifting processes and multiplicative vector fields, Quart. J. Math. Oxford Ser. (2) 49 (1998), no. 193, 59-85.

[10] B. Noohi, On weak maps between 2-groups, arXiv:math/0506313v3 [math.CT].

Department of Mathematics, University of Illinois, Urbana, IL 61801 